\title{
Predictores de satisfacción laboral y bienestar subjetivo en profesionales de la salud. Un estudio con médicos y enfermeros de la ciudad de Rosario
}

Laura Paris•

\section{Resumen}

Se presentan los resultados de una investigación empírica orientada al estudio de los predictores de satisfacción laboral y bienestar subjetivo en profesionales de la salud. Para tal fin, se trabajó con una muestra de 99 enfermeros y 97 médicos quienes respondieron un protocolo integrado por datos sociodemográficos, dos escalas desarrolladas para el presente trabajo (Estresores Asistenciales y Afrontamiento del Estrés), la Escala de Satisfacción Laboral (Shouksmith, 1990) y el Inventario de Bienestar Sujetivo (Nacpal y Shell, 1992). La evidencia indica que los mejores predictores de satisfacción laboral son: la cantidad de horas de trabajo semanal, el afrontamiento de resolución de problemas, la satisfacción con la vida y algunas dimensiones del bienestar tales como la correspondencia entre expectativas y logros, el adecuado manejo mental y el apoyo emocional del grupo familiar. Las diferencias en función de la profesión muestran que, entre los médicos, surgen como predictores el apoyo organizacional y familiar y la coherencia entre expectativas y logros alcanzados (en relación con los salarios y las posibilidades de ascenso). Mientras que, entre los enfermeros, se destaca el papel del afrontamiento cooperativo. Finalmente, los predictores de bienestar subjetivo son: tener hijos, trabajar más horas semanales, percibir justicia organizacional, usar afrontamiento de resolución de problemas y estar satisfecho con el desarrollo de las habilidades y potencialidades. La única diferencia en función de la profesión muestra que los enfermeros responden a los estresores utilizando el distanciamiento emocional y el ocio recreativo con mayor frecuencia que los médicos.

Palabras clave: satisfacción laboral, bienestar, médicos y enfermeros.

Recibido, 27 de octubre de 2010

Aceptado, 21 de marzo de 2011

- Dra. En Psicología. Institución: Universidad Católica Argentina (Facultad de Derecho y Ciencias Sociales del Rosario). Universidad Nacional de Rosario (Facultad de Psicología). 


\title{
Predictors of labor satisfaction an subjective well-being of Health professionals
}

\begin{abstract}
The aim of this study was to analyze the predictors of job satisfaction and subjective well-being in health professionals. We worked with a sample of 99 nurses and 97 doctors from Rosario city (Argentina). The subjects completed a questionnaire of personal data, two scales developed for this study (Care Stressors, and Coping with Care Stress), Job Satisfaction Scale (Shouksmith, 1990), and Subjective Well-being Inventory (Nacpal \& Shell, 1992). Number of weekly working hours, problem-solving coping, satisfaction with life, and some dimensions of well-being (such as correspondence between expectations and achievements, the adequate mental management, and emotional support of family group), emerged as the strongest predictors of work satisfaction. Differences in function of the profession showed that, the best predictors, among physicians, are family and organizational support, and coherence between expectations and achievements (regarding salaries and promotion opportunities). In contrast, among nurses, only highlighted the use of cooperative coping as an explanatory variable. In relation to subjective well-being, the results showed that the best predictors are the following: having children, working more hours per week, perceiving organizational justice, using problem-solving coping, and being satisfied with their development of skills and capacities. The only difference in function of the profession shows that nurses respond to stressors using emotional distance and recreational leisure more often than doctors do.
\end{abstract}

Key words: work satisfaction, well-being, doctors, nurses 


\section{Introducción}

Las profesiones asistenciales se caracterizan por estresores que son el resultado de una tarea de dedicación al cuidado de la vida de los demás, implicando aspectos tales como el contacto con la enfermedad y la muerte, los vínculos con la familia de los pacientes y particularmente en Argentina, estos aspectos se ven agravados por las condiciones propias de nuestro sistema de salud. Las deficiencias de las instituciones sanitarias, tanto en ámbitos públicos como privados colocan a los trabajadores en situaciones de malestar y riesgo permanente. En el ámbito de las instituciones públicas, el recorte de las partidas presupuestarias, la insuficiencia de la infraestructura hospitalaria, la falta de medicamentos y la disminución de los salarios profesionales, configuran un clima laboral inestable y estresante. En el ámbito de las instituciones privadas, por su parte, las condiciones laborales se han ido deteriorando paulatinamente, configurando un ambiente de alta competitividad, que exige una dedicación no equivalente con las retribuciones económicas y sociales percibidas. La responsabilidad de los elevados niveles de estrés que padecen los profesionales de la salud tiene que ver con la exposición constante a estas dificultades, conduciendo a un sentimiento de insatisfacción laboral, que afecta todos los aspectos de la vida del trabajador, impactando profundamente en su bienestar subjetivo.

La satisfacción laboral es interpretada por la mayoría de los autores como un estado emocional o conjunto de sentimientos, resultado de una evaluación cognitiva mediante la que el sujeto compara su realidad cotidiana con un estándar ideal construido a lo largo del tiempo (Tejero-González \& Fernández-Díaz, 2009). Se trata de un constructo muy complejo, ya que varía en función de la motivación y las necesidades personales (Ozel, Bayindir, Zeynep Inan \& Ozel, 2009). Si el resultado de tal comparación es positivo, el sujeto experimentará una sensación de bienestar y satisfacción. En caso contrario, vivenciará elevados niveles de estrés e insatisfacción (Tejero-Gonzalez \& Fernández-Díaz, 2009). Al respecto, Martín García, Luceño Moreno, Jaén Díaz y Rubio Valdehita (2007) han señalado que los trabajadores más estresados tienden a percibir su entorno laboral en forma más adversa y se evalúan como más insatisfechos con algunos aspectos de su trabajo. Asimismo, se han informado relaciones sinérgicas entre insatisfacción y estrés laboral (McClenahan, Giles \& Mallett, 2007), desde el momento que a mayor insatisfacción se han registrado más síntomas de enfermedad.

Recientemente, se ha comenzado a analizar un constructo en el ámbito de la satisfacción con la vida y el bienestar subjetivo que tiene una relevancia potencial para explicar las diferencias transnacionales en satisfacción laboral. Se trata de la positividad (Mueller, Hattrup \& Hausmann, 2009), definida como una variable cultural que refleja la tendencia de los miembros de un grupo para ver la vida "de 
color de rosa" porque valoran los afectos positivos y tienen una visión optimista

de la vida. Sin embargo, la asociación de las dimensiones de bienestar subjetivo con la satisfacción laboral no se han documentado a través de las múltiples facetas del constructo que se han tomado en cuenta en el presente estudio, sino que sólo se han informado vinculaciones acotadas a ciertos aspectos del trabajo, tales como la flexibilidad laboral (Mcnall, Masuda \& Nicklin, 2010), altos salarios y oportunidades de desarrollo de carrera (Zontek, DuVernois \& Ogle, 2009). Con respecto al individuo, la satisfacción laboral general, se ha asociado con un aumento del bienestar (Edwards, Bell, Arthur \& Decuir, 2008), así como con la satisfacción con la vida personal (Mcnall et al., 2010).

Frente a este panorama, la presente investigación se orienta a estudiar los predictores de satisfacción laboral y bienestar subjetivo en médicos y enfermeros de la ciudad de Rosario.

\section{Método}

\section{Participantes}

La verificación empírica se realizó sobre una muestra por disponibilidad integrada por 196 profesionales (97 médicos y 99 enfermeros) de instituciones sanitarias públicas y privadas de la ciudad de Rosario (Argentina). La elección de la muestra se basó en razones de factibilidad y accesibilidad. El 65\% eran mujeres; el promedio de edad de la muestra total fue de 38 años y el promedio de antigüedad laboral de 12 años. En cuanto al tipo de institución, el 32\% trabajaba en instituciones públicas (hospitales, centros de salud), el 40\% pertenecía a privados (clínicas, atención domiciliaria) y el $27 \%$ restante trabajaba en ambos tipos de instituciones.

\section{Instrumentos}

La totalidad de la muestra respondió un cuadernillo integrado por los siguientes instrumentos:

Escala de Estresores Asistenciales. Los estresores asistenciales fueron explorados a través de la escala desarrollada por Paris (2007), integrada por 33 ítems, con formato tipo Likert de 4 puntos $(0=$ nada; $3=$ mucho). La escala explora cinco dimensiones o fuentes de estrés asistencial, a saber: falta de apoyo organizacional (ej.: 'escasa disposición de pares y superiores para integrar equipos de trabajo', $\alpha=0,815$ ); sobrecarga laboral (ej.: 'excesivo número de pacientes a cargo', $\alpha=0,810$ ); dificultades interpersonales (ej.: 'relaciones problemáticas 
con médicos o enfermeros', $\alpha=0,774)$; fuentes extrínsecas de insatisfacción (ej.: 'bajos sueldos' $\alpha=0,748$ ) y falta de justicia organizacional (ej.: 'sensación de dar mucho en el trabajo y no ser recompensado', $\alpha=0,723$ ).

Escala de Estrategias de Afrontamiento del Estrés Asistencial. Las estrategias de afrontamiento fueron evaluadas a través de la escala desarrollada por Paris (2007), conformada por 24 ítems, con formato Likert de 3 puntos $(0=$ nunca; $2=$ siempre). El instrumento explora las siguientes cuatro estrategias de afrontamiento: estrategias desadaptativas (ej.: 'me evado del problema comiendo, fumando o bebiendo', $\alpha=0,880$ ); estrategias de resolución de problemas (ej.: 'planifico cómo resolver el problema', $\alpha=0,757$ ); estrategias de resignación (ej.:'espero que las cosas cambien en algún momento', $\alpha=0,781$ ) y estrategias de distanciamiento (ej.: 'pienso en lo que haré en mi tiempo libre', $\alpha=0,762$ ).

Escala de Bienestar Subjetivo. El bienestar subjetivo fue medido a través de la adaptación argentina (Paris, 2007) de la escala homónima desarrollada por Nacpal \& Shell (1992). Para su validación se siguió el método divulgado por Moraes, Hasselmann y Reichenheim (2002). Vale decir que se efectuó la equivalencia conceptual y de ítems (a través de revisiones bibliográficas sobre el tema y debates con expertos); equivalencia semántica (a través de un proceso de traducción y retraducción); operacional (manteniendo las condiciones de administración en ambos contextos) y de medición (a través del cálculo de coeficientes de confiabilidad y validez). La versión validada quedó integrada por 40 ítems, con formato Likert de 3 puntos (1= casi nunca; $3=$ casi siempre). Si bien la escala original explora 11 factores a través de sus 40 ítems, la versión validada quedó integrada por 10 dimensiones, habida cuenta que los análisis de componentes principales indicaron como la mejor solución la que integraba en un mismo factor las dos primeras dimensiones del instrumento original (bienestar vinculado a afectos positivos y correspondencia entre expectativas y logros). Las dimensiones medidas fueron las siguientes: correspondencia entre expectativas y logros (ej.: '¿piensa que ha logrado el estándar de vida que esperaba?', $\alpha=0,870$ ); confianza en afrontar dificultades (ej.: ' $¿$ siente que puede manejar situaciones inesperadas?', $\alpha=0,700$ ); trascendencia (ej.: ' $i$ ha tenido alguna vez experiencias de intensa felicidad similar al éxtasis?', $\alpha=0,766$ ); apoyo social (ej.: '¿siente que sus amigos y parientes lo ayudarían si estuviera necesitado?', $\alpha=0,853$ ); relaciones con el grupo primario (ej.: ‘¿cómo se siente respecto a la relación con sus hijos?', $\alpha=0,765$ ); inadecuado manejo mental (ej.: ‘¿siente que es fácilmente irritable?', $\alpha=0,843$ ); apoyo del grupo familiar (ej.: ‘ ¿considera que su familia lo ayuda a encontrar soluciones a la mayoría de sus problemas?', $\alpha=0,750$ ); bienestar vinculado a afectos negativos (ej.: ‘¿siente que su vida es inservible?', $\alpha=0,754$ ); 
contactos sociales deficientes (ej.: '¿se preocupa a veces por no mantener una vinculación estrecha con alguien?', $\alpha=0,759)$ y percepción de problemas de salud (ej.: ‘ ¿tiene problemas para dormir?’, $\alpha=0,767$ ).

Escala de Satisfacción Laboral y de Satisfacción con la Vida en General. La satisfacción laboral y con la vida en general fue medida con la adaptación argentina (Paris, 2007) de la escala desarrollada por Shouksmith (1990). En este caso, también se siguió el mismo método de validación utilizado en la adaptación del instrumento anterior. La versión final reprodujo la original y quedó integrada por 12 ítems con 7 opciones de respuesta $(1=$ muy en desacuerdo; $7=$ muy de acuerdo), agrupados en dos dimensiones: satisfacción laboral (ej.: 'mi trabajo me da seguridad laboral', $\alpha=0,807$ ) y satisfacción con la vida en general (ej.: 'mi vida me permite desarrollar todas mis habilidades y potencialidades', $\alpha=0,806$ ).

Variables sociodemográficas. El protocolo de recolección de datos se completó con un conjunto de preguntas orientadas a recabar información acerca de variables sociodemográficas tales como edad, sexo, estado civil, número de hijos, profesión, especialidad (en el caso de los médicos), ámbito laboral en que se desempeña, antigüedad, horas de trabajo semanal, intención de renunciar y desempeño en el ámbito de la docencia.

\section{Procedimiento de recolección de datos}

El contacto con los profesionales se efectuó en los lugares de trabajo habituales, previa solicitud de autorización a las autoridades sanitarias. Luego de hacerles conocer los objetivos del estudio se invitó a los profesionales a formar parte de la investigación. La participación fue voluntaria, previa firma de un consentimiento informado y se tomaron todos los recaudos necesarios para garantizar el anonimato. En cada institución se instalaron urnas especialmente preparadas para que depositaran los protocolos una vez completados. Este mecanismo permitió que cada profesional contestara los cuestionarios en el lugar y momento en que le resultara más conveniente. Durante todo el proceso se respetaron los lineamientos éticos vinculados con la investigación con seres humanos.

\section{Resultados}

Con el propósito de identificar los mejores predictores de satisfacción laboral y bienestar subjetivo se calculó un conjunto de análisis de regresión múltiple. Para la ejecución de dichos análisis se ingresaron, en el primer paso, las variables sociodemográficas a fin de controlar sus posibles efectos sobre la variable dependiente; en el segundo paso, se ingresaron los estresores asistenciales; en 
el tercer paso, las estrategias de afrontamiento del estrés y en el cuarto paso, la percepción de satisfacción con la vida. Tales variables desempeñaron el rol de independientes, en tanto que la satisfacción laboral y el bienestar subjetivo adoptaron el rol de variables dependientes.

Tabla 1 | Predictores de satisfacción laboral en la muestra total

\begin{tabular}{|c|c|c|}
\hline & \\
\hline & \multicolumn{2}{|c|}{ VARIABLE DEPENDIENTE } \\
\hline & \multicolumn{2}{|c|}{ SATISFACCIÓN LABORAL } \\
\hline VARIABLES PREDICTORAS & $\beta$ estandarizada & $F$ \\
\hline \multicolumn{3}{|l|}{ Primer paso (variables sociodemográficas) } \\
\hline \multirow{2}{*}{$\begin{array}{l}\text { Intención de permanecer } \\
\text { Horas }\end{array}$} & $.265^{\star \star \star}$ & \\
\hline & $.213^{\star \star}$ & \\
\hline \multicolumn{2}{|l|}{$\mathrm{R}^{2}=.124$} & \\
\hline \multicolumn{2}{|l|}{$\Delta \mathrm{R}^{2}=.056$} & $F(14,181)=1.825^{*}$ \\
\hline \multicolumn{3}{|l|}{ Segundo paso (estresores) } \\
\hline \multirow{3}{*}{$\begin{array}{l}\text { Fuentes extrínsecas de insatisfacción } \\
\text { Falta de apoyo organizacional } \\
\text { Dificultades interpersonales }\end{array}$} & $-.400^{\star \star \star}$ & \\
\hline & $-.301^{\star \star \star}$ & \\
\hline & $-.185^{\star \star}$ & \\
\hline \multicolumn{3}{|l|}{$\mathrm{R}^{2}=.500$} \\
\hline \multicolumn{2}{|l|}{$\Delta \mathrm{R}^{2}=.446$} & $F(19,176)=9.264^{\star \star \star}$ \\
\hline \multicolumn{3}{|l|}{ Tercer paso (afrontamiento) } \\
\hline Resolución de problemas & $.176^{\star}$ & \\
\hline \multicolumn{2}{|l|}{$\mathrm{R}^{2}=.543$} & \\
\hline \multicolumn{2}{|l|}{$\Delta \mathrm{R} 2=.482$} & $F(23,172)=8.888^{\star \star \star}$ \\
\hline \multicolumn{3}{|l|}{ Cuarto paso (satisfacción con la vida) } \\
\hline Satisfacción con la vida & $.234^{\star \star}$ & \\
\hline \multicolumn{3}{|l|}{$\mathrm{R}^{2}=.575$} \\
\hline \multicolumn{2}{|l|}{$\Delta \mathrm{R}^{2}=.515$} & $F(24,171)=9.641^{\star \star \star}$ \\
\hline \multicolumn{3}{|l|}{ Quinto paso (bienestar subjetivo) } \\
\hline \multirow{3}{*}{$\begin{array}{l}\text { Correspondencia expectativas y logros } \\
\text { Manejo mental } \\
\text { Apoyo del grupo familiar }\end{array}$} & $.216^{\star \star}$ & \\
\hline & $.204^{\star \star}$ & \\
\hline & $.140^{\star}$ & \\
\hline \multicolumn{3}{|l|}{$\mathrm{R}^{2}=.625$} \\
\hline$\Delta R^{2}=.545$ & & $F(34,161)=7.880^{\star \star \star}$ \\
\hline
\end{tabular}

${ }^{*} p>0.05 ;{ }^{* \star} p>0.01 ;{ }^{* \star \star} p>0.001$ 
En total, las variables predictoras explican un $54.5 \%$ de la varianza de la satisfacción laboral. Los valores (y signos) de los coeficientes $\beta$ estandarizados indican que las variables horas de trabajo, intención de permanecer en el puesto de trabajo, el afrontamiento de resolución de problemas, la satisfacción con la vida y la correspondencia entre expectativas y logros, el adecuado manejo mental y el apoyo percibido por parte del grupo familiar, son los mejores predictores de la satisfacción laboral entre los trabajadores de la salud (médicos y enfermeros). En tanto que, algunos estresores asistenciales, tales como fuentes extrínsecas de insatisfacción, falta de apoyo organizacional y dificultades interpersonales (dados los signos negativos de los correspondientes coeficientes $\beta$ estandarizados), surgen como los predictores más idóneos de la insatisfacción laboral. En la Figura 1 se puede observar el aporte de cada variable a la explicación de la satisfacción laboral:

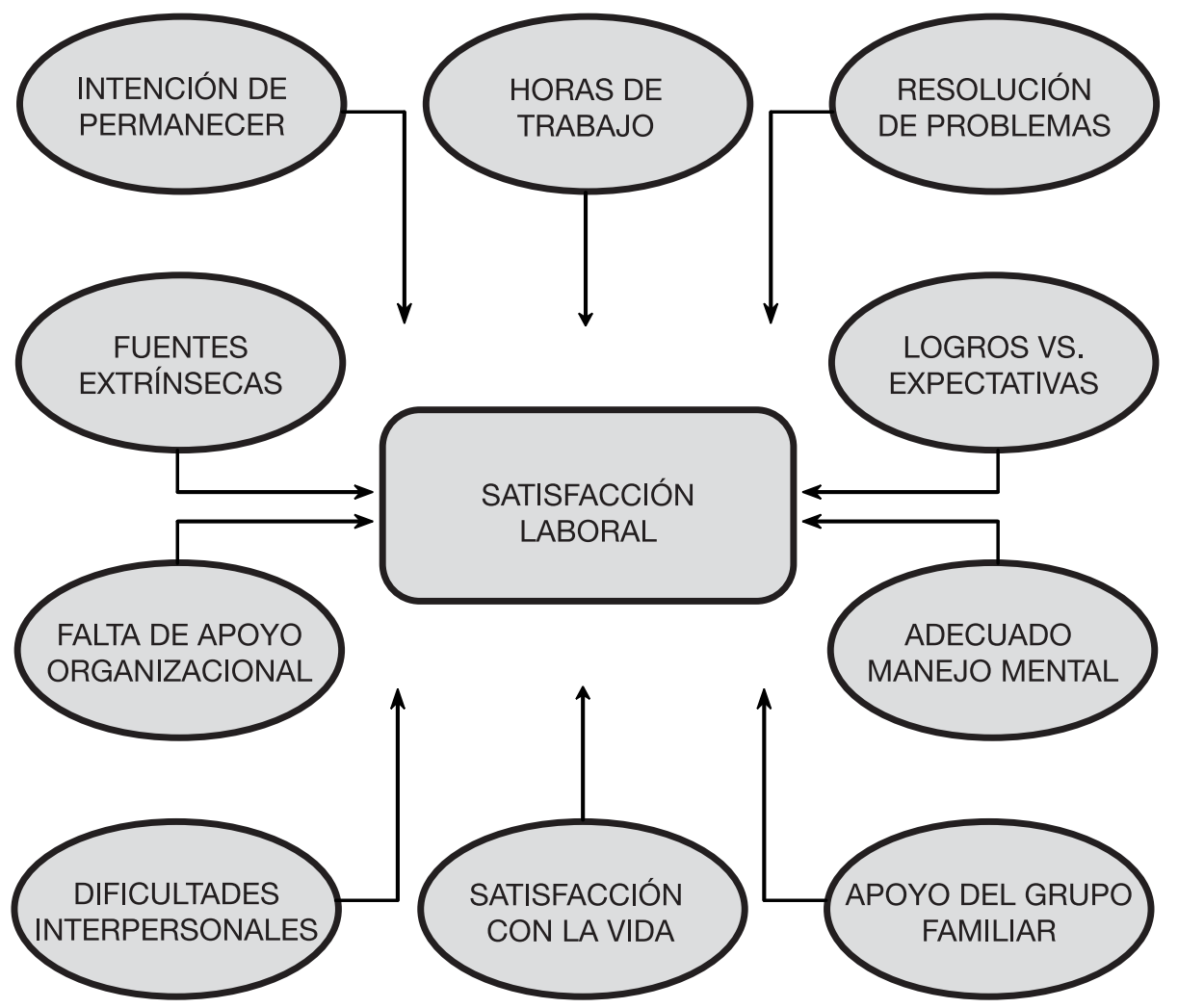

Figura 1 | Predictores de satisfacción laboral en la muestra total

Seguidamente, a efectos de explorar las diferencias en función de la profesión, se analizó por separado los predictores de satisfacción en médicos y enfermeros. Los resultados obtenidos muestran que, entre los médicos, surgen como los 
mejores predictores el apoyo organizacional y familiar y la coherencia entre expectativas y logros alcanzados (en relación con los salarios y las posibilidades de ascenso). Mientras que, entre los enfermeros, se destaca el papel del afrontamiento cooperativo y la resolución directa de los problemas laborales, ya sea a través de la planificación de una estrategia para solucionarlos o de la puesta en marcha de un plan de acción inmediato.

Con idéntica metodología se analizaron las variables independientes que mejor explicaban el bienestar subjetivo entre los profesionales de la salud en general.

\section{Tabla 2 | Predictores de bienestar subjetivo en la muestra total}

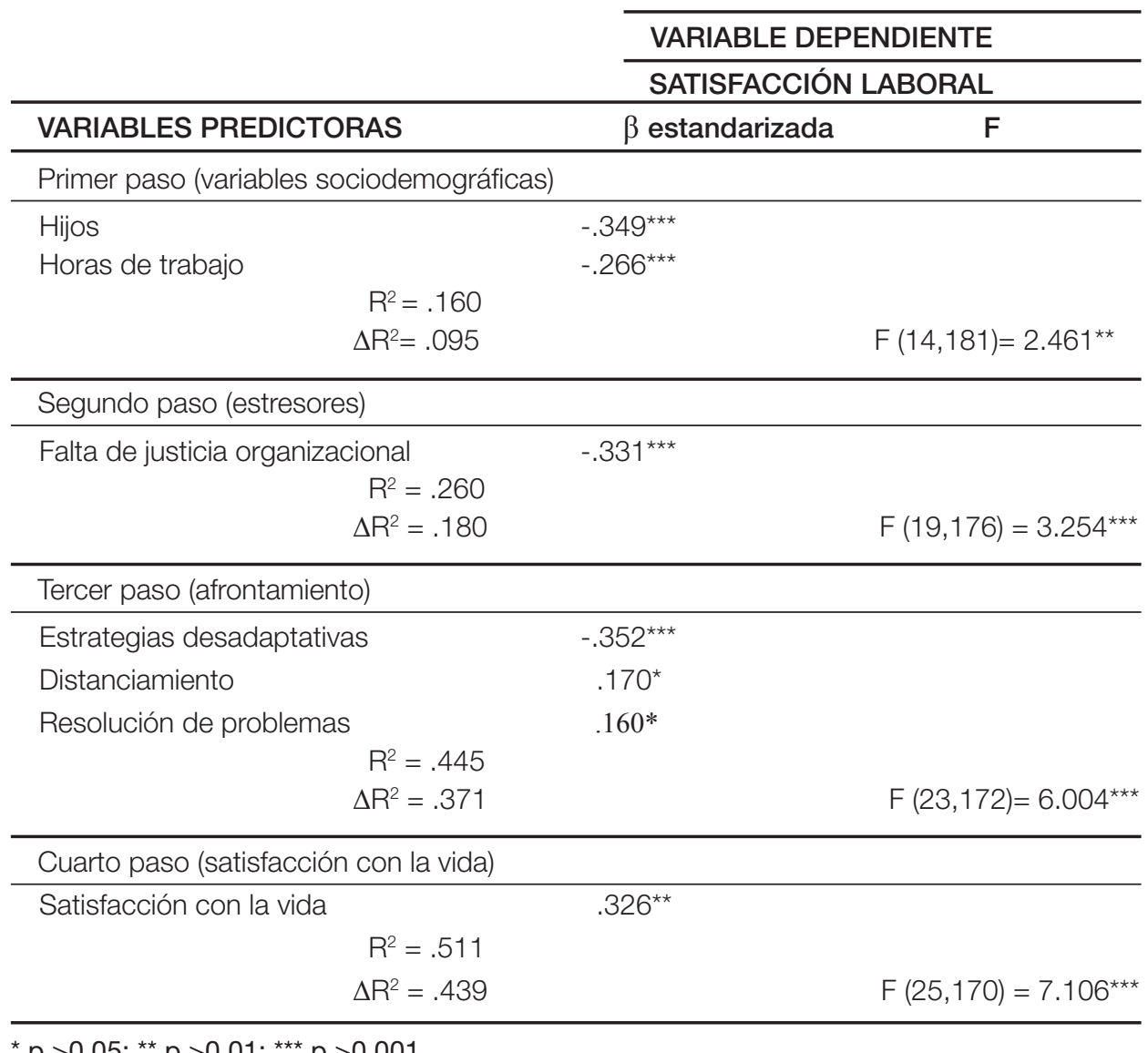

Las variables predictoras, en total, explicaron el $43.9 \%$ de la varianza del bienestar subjetivo y los coeficientes $\beta$ estandarizados indican que los profesionales que tienen más bienestar subjetivo, son los que tienen hijos, trabajan más horas semanales, perciben más justicia organizacional, usan 
afrontamiento de resolución de problemas y distanciamiento, no utilizan el afrontamiento desadaptativo y están satisfechos con su vida.

Nuevamente se analizaron las diferencias en bienestar en función de la profesión (médicos y enfermeros), observándose un patrón común entre ambos profesionales. La única diferencia radica en que los enfermeros responden a los estresores utilizando el distanciamiento emocional (logrando, por ejemplo, una mayor separación operativa entre el trabajo y la vida personal) y el ocio recreativo con mayor frecuencia que los médicos, lo que se traduce en un mayor bienestar subjetivo.

\section{Consideraciones Finales}

La presente investigación se ha desarrollado dentro del marco teórico provisto por la teoría transaccional del estrés de Lazarus y Folkman (1984). Desde esta perspectiva, la experiencia del estrés es una construcción de naturaleza predominantemente subjetiva desde el momento que los individuos movilizan tanto factores personales como situacionales para evaluar el potencial perjudicial de los eventos. Cuando el sujeto siente que no cuenta con los recursos o estrategias suficientes para afrontar las demandas que percibe como estresantes, experimenta diferentes niveles de estrés que pueden llegar a vulnerar su equilibrio y bienestar psicológicos. Si, además, el estrés es vivenciado en el ámbito laboral, puede repercutir sobre la calidad de su trabajo, el nivel de compromiso organizacional y su grado de satisfacción laboral-personal.

Con base en tales consideraciones, el objetivo del presente estudio estuvo orientado a identificar los predictores de satisfacción y bienestar subjetivo entre los médicos y enfermeros que desempeñan su trabajo en el ámbito de ciudad de Rosario. La intención de permanecer en el puesto de trabajo, el afrontamiento de resolución de problemas, la satisfacción con la vida y algunas dimensiones del bienestar (tales como la correspondencia entre expectativas y logros, el adecuado manejo mental y el apoyo percibido por parte del grupo familiar), emergieron como los mejores predictores de la satisfacción laboral asistencial. En tanto que los aspectos extrínsecos al trabajo, la falta de apoyo organizacional y las dificultades interpersonales, surgieron como los predictores más fuertes de la insatisfacción laboral. Similares resultados han sido reseñados en la bibliografía especializada (McGillis Hall \& Doran, 2007; Yang, 2009; Zontek et al., 2009), lo que muestra que más allá de las diferencias culturales, hay aspectos de la vida laboral que trascienden los contextos sociales y políticos, locales o regionales. Al 
analizar los determinantes de la satisfacción laboral en función de la profesión, se pudo observar que la percepción de apoyo organizacional y familiar, la coherencia entre expectativas y logros, y las mayores posibilidades de ascenso y promociones, son los principales predictores de satisfacción entre los médicos. En tanto que los enfermeros más satisfechos con su trabajo son aquellos que tienen intenciones de permanecer en su puesto de trabajo, perciben apoyo laboral, resuelven los problemas de manera activa y cooperativa, tienen un adecuado manejo mental y gozan de buena salud.

En cuanto a la predicción del bienestar subjetivo, los resultados obtenidos a partir de la muestra total indican que las variables con mayor poder explicativo son tener hijos, trabajar más horas semanales, percibir justicia organizacional, usar afrontamiento de resolución de problemas y de distanciamiento, y estar satisfecho con la posibilidad de desarrollar las propias habilidades y potencialidades. Al diferenciar entre médicos y enfermeros, la evidencia reunida indica que los médicos que experimentan mayor bienestar subjetivo son aquellos que perciben justicia en las retribuciones y en el trato interpersonal, trabajan muchas horas semanales aunque concentran sus esfuerzos en un solo empleo, tienen hijos y responden al estrés con estrategias adaptativas. Este conjunto de variables, a su vez, caracteriza el bienestar de los enfermeros, con el agregado que este grupo ocupacional responde a los estresores utilizando el distanciamiento emocional y el ocio recreativo con mayor frecuencia que los médicos.

\section{Fortalezas, limitaciones y sugerencias para futuros estudios}

Como toda investigación empírica, el presente estudio se caracteriza por algunas debilidades y fortalezas. Entre las debilidades hay que remarcar la composición de la muestra en estudio, ya que por haber estado integrada por disponibilidad impediría la generalización de los resultados a toda la población de profesionales de la salud argentinos. No obstante y, en sintonía con lo puntualizado por un gran número de investigadores en el área (Marradi, Archenti \& Piovani, 2007; Skytt, Ljunggren \& Carlsson, 2007), como en la composición de la muestra se incluyeron representantes de las más diversas instituciones asistenciales del medio, los resultados obtenidos podrían considerarse un fiel reflejo de la situación asistencial local. Otra limitación del estudio podría estar referida al carácter auto descriptivo de la mayoría de los instrumentos empleados para la recolección de los datos. Aspecto que podría haber generado tanto sesgos derivados de la varianza del método común como una mayor tendencia a la deseabilidad social. En este sentido, los participantes pueden haber ofrecido respuestas movilizadas por el deseo de presentar una imagen mejorada de sí mismos, ocultando o dejando de reconocer algunos síntomas sociales y/o laboralmente mal vistos. Sin embargo, 
previendo tales contingencias, se tomaron todos los recaudos necesarios para garantizar el carácter anónimo no sólo del protocolo sino también del proceso de devolución de los formularios completados, evitando que jefes y supervisores pudieran tener acceso a la información suministrada por sus subalternos. Este último aspecto se vincula a lo que podría constituir una tercera limitación de este estudio, ya que el proceso de recolección de datos demandó casi seis meses debido a las dilaciones y retrasos en la devolución de los cuestionarios.

Entre las fortalezas, hay que destacar el empleo de instrumentos de naturaleza émica que contribuyen a aumentar la validez y confiabilidad de los hallazgos (Briner, Harris \& Daniels, 2004; Dewe, 2004). Los dos instrumentos desarrollados para esta ocasión (Escalas de Estresores Asistenciales y de Estrategias de Afrontamiento del Estrés Asistencial), basados en las características idiosincráticas de la población objetivo, posiblemente han permitido captar más apropiadamente el significado connotativo de los constructos investigados y la intensidad y dirección de sus interrelaciones. Por sus características y propiedades psicométricas, se trata de instrumentos que podrían reemplazar a los utilizados hasta el momento, los que por provenir de contextos laborales foráneos quizá no llegaban a captar todos los matices de la problemática local.

A la luz de los resultados obtenidos, y como orientación para futuras investigaciones, se sugiere profundizar el estudio de la satisfacción laboral y el bienestar en relación con las fuentes disposicionales y particularmente con la taxonomia de autoevaluación de Judge y Bono (2001, citados por Judge, Heller \& Klinger, 2008) dada la relevancia de rasgos tales como auto eficacia y estabilidad emocional en la problemática. Por otra parte, en futuros estudios se podría analizar el impacto de los comportamientos extra-papel (Omar, 2010) sobre el bienestar y la satisfacción laboral asistencial. Sobre todo porque el trabajo propio de algunos departamentos hospitalarios (urgencias, cirugías y similares) se asienta en la ayuda y la colaboración permanente entre los profesionales. Es posible que si perciben justicia, se involucren con más frecuencia en la ejecución de comportamientos extra-papel positivos (tales como altruismo, cortesía, lealtad y similares). Como también es posible que si perciben injusticias, protagonicen comportamientos extra-papel negativos (tales como acoso, revancha, represalia, y similares), los que, a la postre, pueden repercutir sobre el cuidado y atención de los propios pacientes.

Como corolario del trabajo realizado, se sugiere poner en marcha programas de intervención para incrementar la satisfacción laboral y el bienestar en los servicios de salud. Tales programas podrían consistir tanto en el entrenamiento en estrategias para afrontar eficazmente el estrés, como en la implementación regular de espacios grupales de diálogo que favorezcan el desarrollo del apoyo interpersonal. Estas instancias de interacción podrían cumplir un rol preventivo, 
anticipando la aparición de trastornos sociopsicológicos que erosionan el bienestar y la satisfacción personal, al tiempo que contribuirían a crear una atmósfera laboral atravesada por el compromiso de mejorar continuamente.

\section{Bibliografia}

Briner R, Harris C, Daniels K. (2004). How do work stress and coping work? Toward a fundamental theoretical reappraisal. British Journal of Guidance \& Counseling, 32(2), 223-234.

Dewe P. (2004). Work stress and coping: theory, research and practice. British Journal of Guidance \& Counseling, 32(2), 139-142.

Edwards, B.D., Bell, S.T., Arthur, W. \& Decuir, A.D. (2008). Relationships between facets of job satisfaction and task and contextual performance. Applied Psychology: an International Review, 57, 441-465.

Judge, T.A., Heller, D. \& Klinger, R. (2008). The dispositional sources of job satisfaction: a comparative test. Applied Psychology: an International Review, 57, 361-372.

Lazarus, R. \& Folkman, S. (1984). Stress, appraisal and coping. New York: Springer Publishing Company.

Marradi, A.; Archenti, N.; Piovani, J.I. (2007). Metodología de las ciencias sociales. Buenos Aires: Emecé.

Martín García, J., Luceño Moreno, L., Jaén Díaz, M. \& Rubio Valdehita, S. (2007). Relación entre factores psicosociales adversos, evaluados a través del cuestionario Decore y salud laboral deficiente. Psicothema, 19, 95-101.

McClenahan, C., Giles, M. \& Mallett, J. (2007). The importance of context specifity in work stress research: A test of the Demand-Control-Support model in academics. Work \& Stress, 21, 85-95.

McGillis Hall, L. \& Doran, D. (2007). Nurses' perceptions of hospital work environments. Journal of Nursing Management, 15, 264- 273.

Mcnall, L.A., Masuda, A.D. \& Nicklin, J.M. (2010). Flexible work arrangements, job satisfaction and turnover intentions: the mediating role of work-to-family enrichment. The Journal of Psychology, 144, 61-81.

Moraes, C., Hasselmann, M. \& Reichenheim, M. (2002). Adaptaçào trascultural do instrumentos. Cadernos do Saùde Pùblica, 18, 163-176. 
Mueller, K., Hattrup, K. \& Hausmann, N. (2009). An investigation of cross-national differences in positivity and job satisfaction. Journal of Occupational and Organizational Psychology, 82, 551-573.

Nacpal, A. \& Shell, B. (1992). Subjective Well-being Inventory. Nueva Delhi: OMS.

Omar, A. (2010). Comportamientos extra papel. De la colaboración a la transgresión. Revista Interamericana de Psicología Ocupacional, 29, 1, 32-53.

Ozel, A., Bayindir, N., Zeynep Inan, H. \& Ozel, E. (2009). The effect of educational differences on the level of job satisfaction in police officers in Turkey. International Journal of Police Science and Management, 11, 358-365.

Paris, L. (2007). Estrés laboral asistencial, recursos de afrontamiento y satisfacción laboral en trabajadores de la salud de la ciudad de Rosario. Tesis Doctoral en Psicología inédita. Facultad de Psicología, Universidad Nacional de Rosario, Rosario.

Shouksmith, G. (1990). A construct validation of a scale for measuring work motivation. New Zealand Journal of Psychology, 18, 76-81.

Skytt, B., Ljunggren, B. \& Carlsson, M. (2007). Reasons to leave: the motives of first-line nurse managers'to leaving their posts. Journal of Nursing Management, 15, 294-302.

Tejero-Gonzalez, C.M. \& Fernández-Díaz, M.J. (2009). Medición de la satisfacción laboral en la dirección escolar. Relieve, 15, 1-16.

Yang, Y.F. (2009). An investigation of group interaction functioning stimulated by transformational leadership on employee intrinsic and extrinsic job satisfaction: an extension of the resource-based theory perspective. Social, Behavior and Personality, 37, 1259-1278.

Zontek, TL., DuVernois, C.C. \& Ogle, B.R. (2009). Job satisfaction and issues related to the retention of environmental health professionals in North Carolina. Journal of Environmental Health, 72,10-15. 